great difficulty and uncertainty, owing to the position of the gut. Being strongly led to suspect that the intes. tines communicated with the bladder, I did not deem it justifiable. It is worthy of notice that this child lived nine days.

\section{FIBROID INFILTRATION OF THE STOMACH, CONSEQUENT ON CHRONIC GASTRITIS.}

By Percy Bourton, M.D., Beverley.

E. MI., aged 22, had led a most immoral life, and had gonorrhœa three times before she was fourteen years of age. Three and a half years ago, after confinement (her first and last), she began to complain of a burning pain at the region of the stomach, and was treated by a dispensary doctor, evidently for acute gastritis, most probably brought on by the excessive use of raw spirits, for which she had from her youth had a great predilection. This had run on to the chronic form, partly from the more frequent use of ardent spirit to deaden the pain, and partly from neglect; and thus matters had gove on gradually getting worse, till last February.

Between last February and the 6th of October, the dis. pensary doctor had, at intervals, prescribed either hydrocyanic acid to relieve sickness, or astringents for the concomitant diarrhœa; but since the first attack (three and a half years ago) nothing had been done towards allay. ing the chronic gastritis.

On Monday, the 6th October, the mother came in a great hurry for me, thinking that her daughter was dying, and wished me to examine her (which had never before been done), and to tell her disease and present state.

I found her dreadfully emaciated; the limbs were slightly œdematous; and she was too weak to speak but in a whisper. Her pulse was imperceptible, or nearly so. I ascertained that she was not pregnant, and that the catamenia had appeared at the usual time, and in the usual quantity, etc., about a week previous. She complained of great pain at the region of the stomach; and, on examination, a tumour, as large as a cricket-ball, was felt, hard and tender on pressure.

I found that everything which was taken was vomited instantly with great force; coffee-ground vomit accornpanying, and some times pure blood alone. In consequence of the vomiting, no food had been kept on the stomach for many days. It was evident at once, that a hypertrophied and hardened stomach was the cause of these appearances and symptoms; and, considering her very young to have cancer of the stomach, 1 told her friends that it was a disease resembling cancer of the stomach, but not cancer-meaning the cirrhotic inflammation of Dr. Brinton.

She lingered on for one week, during which time I ordered mustard or fomentations to the parts externally. As nothing stayed on the stomach, I ordered food and laudanum per anum, and tried to settle the stomach with ice, chloroform, opium, etc.; but all to no use, and she died on Monday the 13th October.

I got leave to make an opening in the abdomen to examine the stomach, and found the following appear. ances.

The stomach, immediately on opening the peritoneum, appeared as a more or less purple livid tumour, as large as a good sized cocoa-nut. After dissecting for some time to separate it from the surrounding parts, I found that it was so adherent to a hob-nail liver, the diaphragm, etc., that I should have to remove far more than I expected, in order to get the stomach entire, and more than the friends wished; I therefore made an incision in to the viscus, when there followed an immediate flow of watery fluid and collapse of the organ. I then discovered a perfect cast of the stomach of a gelatinous consistence, lying, aiso collapsed, inside the stomach. This curious cast looked exactly as if made of hard-boiled white of egg, and was very like it in every respect. Unless it were क्षे diseased and hypertrophied mucous membrane, it was perfect abnormality ; for after removing it entire, tho. stomach itself inside was covered with a thick layer of fibro-cartilaginous material-white, uneven and corrodedes thicker at some parts than at others; in fact, represent ing exactly what one would expect in a case of fibroid in filtration consequent on chronic gastritis, or what $\mathrm{Dr}$ 근 Brinton has called the "cirrhotic inflammation." ff should think so diseased a stomach has rarely been seen in so young a subject before.

\section{RARE CASES IN MIDWIFERY.}

\section{By Edward Copeman, M.D., Physician to the Norfoll and Norwich Hospital. \\ [Continued from page 507.]

Case xvirr. Puerperal Disease. On July 18th, I waș summoned to a distance to see a lady who had been confined several days. She had had several children before and on two occasions her labours had been followed bye an attack of phlegmasia dolens. On the present occasion she had a chill before labour, but went on well for a dayb or two. Then another chill occurred, followed by greats depression, and other symptoms indicative of poisoned blood; the lochia were one day very offensive, and then almost ceased. She became intolerant of light and noise and it was with great difficulty she could be kept from fainting. Her pulse rose to 120, and was feeble; her respiration was panting or sighing, and her mind slightlyo wandered. The abdomen was distended, but not painfu巴 on pressure. Her surgeon had very judiciously got thebowels open, and applied turpentine stupes to the abdoo men; and when I arrived in the afternoon of the 10 she was better than shelhad been in the morning, beth patient and surgeon attributing the amendment to turpentine. The pulse was down a little below 100, her mind was tolerably clear, although her aspect wat still unfavourable, and she appeared much exhaustedo We agreed to persevere with the turpentine externallyo and also to give it internally should there be any increase of the symptoms; but not otherwise, as she expressed great repugnance to it and felt sure it would make hes? sick. We also allowed wine freely, and ordered a turpen? tine enema if anything were required for the bowels. The vagina to be thoroughly washed out daily.

In conversation, $\mathrm{Mr}$. - expressed equal confidence in the turpentine treatment with myself, and said he had not lost a case of puerperal fever since he employed it.

August 7th. He writes, "Our patient has been pro: gressing favourably since your visit, but her tongue re 3 mains coated in the middle with a dark fur, which has increased since you saw her. In every other respect, her recovery is perfect." Again on the 10th, in answer toె further inquiries, "There was no occasion to give turpen tine internally, nor even by enema; it was only applied to the abdomen for two or three days after you sain her."

Case xix. Severe Puerperal Disease. On Friday December 7 th, 1860, I was requested by Mr. a robust florid Irishwoman, the wife of a small farmer and dealer; but, although of strong and healthy appear ance, some doubt had been expressed as to the conditions of her lungs; and two or three of her brothers, also fing looking men, had quickly succumbed under illness. Sh was confined the Monday before; labour was natural and not prolonged; but for some days previously she had crampy pains in the uterus, sufficiently severe to requir ${ }^{+}$ opiates to subdue them. On Tuesday evening she had a0 chill; and was restless in the night, having at times severe pains in the abdomen. Mr. - gave a dose of six drachmo of castor oil and half an ounce of oil of turpentine, and opiates afterwards. On Wednesday, the lochial discharg 